\section{Noninvasive vascular imaging in fluorescent tumors using multispectral unmixing}

\author{
Patrick A. Mayes, David T. Dicker, Yingqiu Y. Liu, and Wafik S. El-Deiry \\ Laboratory of Molecular Oncology and Cell Cycle Regulation, Departments of \\ Medicine (Hematology/Oncology), Genetics, and Pharmacology, the Institute for \\ Translational Medicine and Therapeutics, and the Abramson Comprehensive Cancer \\ Center, University of Pennsylvania School of Medicine, Philadelphia, PA, USA
}

BioTechniques 45:459-464 (October 2008)

doi 10.2144/000112946

\begin{abstract}
Noninvasive imaging of tumor vascularization in animal models provides an important tool for studying the biology of tumor angiogenesis as well as monitoring the effects of antiangiogenic therapies. Through the use of in vivo multispectral fluorescent imaging, we have discovered a distinct spectral signature associated with blood vessels present in fluorescent tumors in mice. This unique spectral signature allows for the tumor vasculature to be imaged and quantified without the use of vascular imaging probes. This noninvasive vascular imaging technique allows for real-time analysis of tumor vascularization, which provides a powerful and efficient tool for monitoring the effect of antiangiogenic therapies in preclinical animal models.
\end{abstract}

The growth of tumors depends on the formation and maintenance of a vascular supply. In response to low oxygen conditions within the tumor microenvironment, tumor cells stimulate the process of tumor vascularization through the secretion of proangiogenic factors (1). This principle has led to the development of anti-cancer therapies that inhibit tumor angiogenesis (2). However, much still needs to be learned about the biological process of tumor angiogenesis and how best to inhibit it to provide the greatest therapeutic effect.

A number of vascular imaging probes have been developed that allow for the noninvasive detection of blood vessels in live animals and embryos using fluorescent imaging. Current vascular imaging probes include fluorescently labeled macromolecules $(3,4)$, liposomes (5), nanoparticles $(6,7)$, and quantum dots (8). There are specific advantages and disadvantages related to cost, toxicity, bioavailability, and stability for each of these probes. All of these agents can be categorized as "blood-pooling probes," whereby they cannot permeate intact vasculature, thus providing the specificity required for vascular imaging. However, these vascular probes have a significant disadvantage for use in detecting the vascularization of tumors.
Unlike the hierarchically organized vasculature of normal tissue, tumors form highly irregular and often immature blood vessels resulting in inconsistent blood flow and poor vascular integrity (9). This hallmark of tumor vasculature causes bloodpooling vascular imaging probes to leak from tumor vasculature and selectively accumulate in solid tumors. This results in an artificially high and prolonged probe signal within tumors, independent of the density of tumor vasculature. Many probes with a long half-life show tumor-specific signal for weeks following the initial intravenous injection $(10,11)$. This suggests that the high intratumoral signal detected with blood-pooling vascular imaging probes is, at least in part, an artifact of vessel leakiness and a lack of probe clearance from within tumors. Reports have shown that if imaging of intravascular probes is done acutely following intravenous injection $(\leq 30 \mathrm{~min})$, minimal leakage will occur (12), which provides suitable vascular contrast and specificity for initial imaging. However, subsequent imaging can only be carried out after the tumor's complete clearance of previous probe injections. This property of blood-pooling probes makes them unsuitable for monitoring dynamic changes in the tumor vasculature of animal models during tumor growth as well as in response to antiangiogenic therapy.

Here we describe a technique to detect and monitor dynamic changes in the tumor vasculature of living animals without the use of vascular imaging probes. Using commercially available multispectral imaging technology, we were able to simultaneously image a range of wavelengths. This multiplexing capability allows differentiation and unmixing of spectral differences as small as $10 \mathrm{~nm}$ from a single image. Using this technology, we have detected and quantified a distinct spectral emission signature of blood vessels present in fluorescent tumors.

One million HCT116 p53-null colon carcinoma cells with stable expression of DsRed protein (RFP) (Clontech, Mountainview, CA, USA) were mixed 1:1 with 50\% Matrigel Matrix (BD Biosciences, San Jose, CA, USA) and injected subcutaneously into the rear flanks of nude mice (The Jackson Laboratory, Bar Harbor, ME, USA). After three weeks, tumors were $1-2 \mathrm{~cm}$. Mice were anesthetized with ketamine/xylazine and imaged using either the Maestro in vivo fluorescent imager with a Xenon light source (CRi, Inc., Woburn, MA, USA) or the Nuance camera (CRi, Inc.) attached to an AZ100 fluorescence dissecting microscope with a mercury light source (Nikon, Melville, NY, USA).

The manufacturers CRi and Nikon recommended filter sets for imaging RFP with the Maestro and Nuance/ AZ100 setup, respectively. 530/50 nm excitation and $580 \mathrm{~nm}$ longpass filters were used for the Maestro, and 470/40 $\mathrm{nm}$ excitation and $495 \mathrm{~nm}$ longpass filters were used for the Nuance/AZ100 setup. The multispectral acquisition range was $580-800 \mathrm{~nm}$ in $10-\mathrm{nm}$ steps. Following image acquisition we used the spectral processing feature of the CRi software to mark an area of the tumor where a large blood vessel was visualized by the naked eye and saved the spectral emission signature as a red pseudocolor in our spectral library (Figure 1A). We then marked an area of the tumor where no blood vessel was visually apparent and saved the spectral emission signature as a green pseudocolor in our spectral library (Figure 1A). The spectral emission signatures of the two areas were nearly identical except that the vascular signature (red 


\section{Benchmarks}

pseudocolor) exhibited a 10-nm upward shift in wavelength. The saved spectral signatures were then unmixed and mapped across the bitmap of acquired tumor images. We presume the increase in wavelength corresponds to either a decrease in the frequency, or the scattering of fluorescent light emitted from tumor cells as it passes through blood vessels present within the tumor. Therefore, fluorescent light is derived from the tumor cells but altered by the presence of blood vessels between the tumor cells and the detection source.

With multispectral unmixing we identified blood vessels present throughout tumor images taken at $1 \times$ magnification using the Nuance/ AZ100 setup (Figure 1B). At increased (8.7×) magnification we observed capillaries of approximately $10 \mu \mathrm{m}$ in diameter growing throughout the tumor (Figure 1C), illustrating the sensitivity of this method of vascular imaging. Depth penetration of multispectral vascular imaging is determined by the wavelength as well as the brightness of the endogenous fluorophore being expressed within the tumor cells. We also observed a decrease in the depth penetration of vascular imaging as the magnification was increased. Therefore, the deeper the blood vessel is located, the bigger it must be to be
B
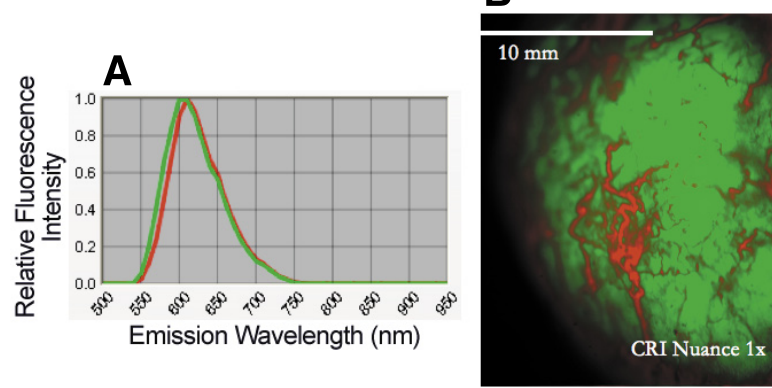

C

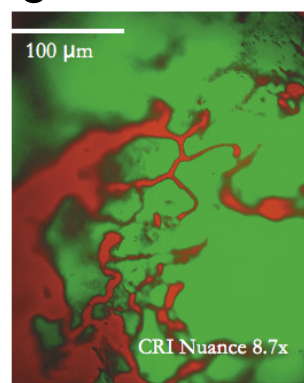

D

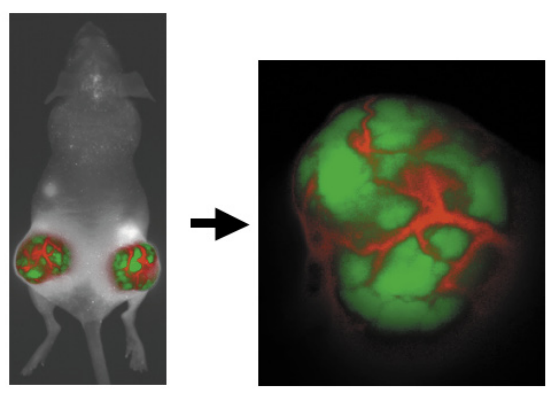

Vascular Component Image

E

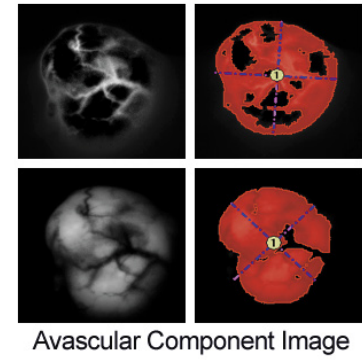

Figure 1. Noninvasive imaging of tumor vasculature using multispectral unmixing in fluorescent tumors. (A) Pixels of the bitmap where emitted fluorescence has passed through a blood vessel have a spectral emission signature peak at $620 \mathrm{~nm}$ (red spectra). Pixels present in avascular areas of the tumor have a spectral emission signature peak at $610 \mathrm{~nm}$ (green spectra). The two independent spectral emission signatures can be saved and mapped in pseudocolor on each image. (B) An image of a fluorescent tumor taken using the CRi Nuance camera attached to an AZ100 dissecting microscope from Nikon. (C) A high magnification image of the same tumor area as (B) showing the ability to detect capillaries (5-10 $\mu \mathrm{m}$ in diameter) within fluorescent tumors using multispectral unmixing. (D) Multispectral unmixing of a macroscopic whole body image of a mouse with fluorescent tumors on both rear flanks using the CRi setup (left panel). Enlarged image of the left rear flank tumor. All auto-fluorescence from the skin and other organs was unmixed and removed from the image (right panel). (E) Using the CRi software, a custom-threshold region of interest (ROI) is created in the vascular and avascular component images. A vascular index is then calculated by dividing the avascular component total area $\left(\mathrm{mm}^{2}\right)$ by the vascular component total area $\left(\mathrm{mm}^{2}\right)$.
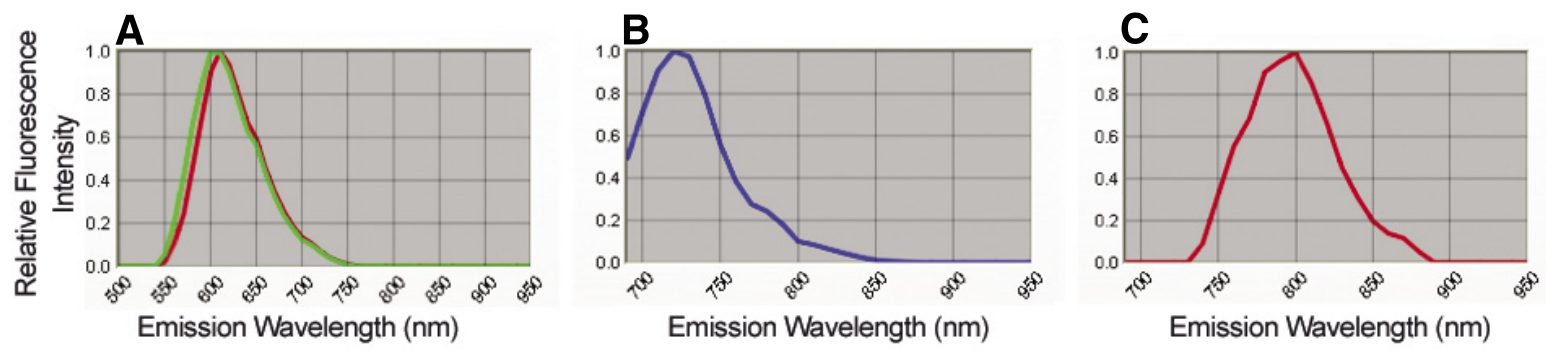

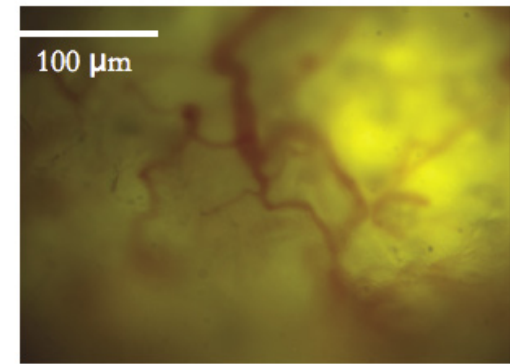

Multispectral Unmixing

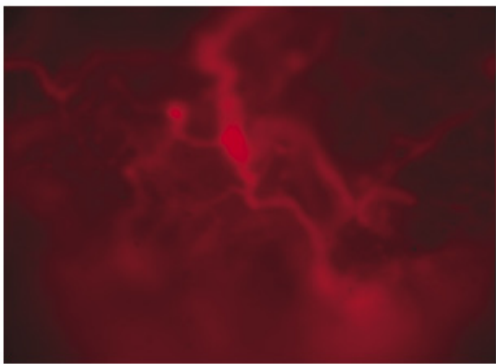

Angiosense $680 \mathrm{~nm}$

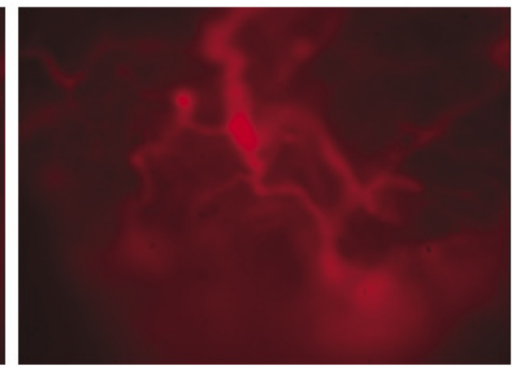

Q-tracker $800 \mathrm{~nm}$

Figure 2. Imaging tumor vasculature with multispectral unmixing has comparable sensitivity to two commercially available vascular imaging probes, Angiosense $680 \mathrm{~nm}$ and Q-Tracker $800 \mathrm{~nm}$. (A) Multispectral unmixing of the endogenous RFP expression from tumors detects blood vessels present throughout the tumor area. (B) Vascular imaging using Angiosense $680 \mathrm{~nm}$ probe or (C) Q-Tracker $800 \mathrm{~nm}$ probe detects the same blood vessels detected via multispectral unmixing. The three panels are independent images taken of the same tumor area using the respective filter sets. The graphs for each panel represent the spectral emission signature for each respective vascular imaging technique. Images were taken using the CRi Nuance camera attached to the AZ100 dissecting microscope from Nikon. 


\section{Three Steps to Faster Cell Disruption}

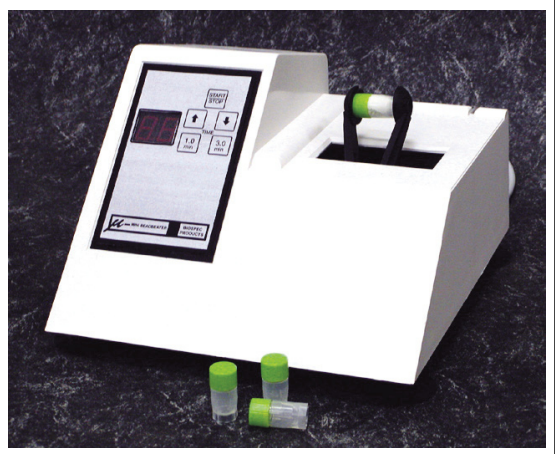

Robust mechanical grinding significantly enhances cell disruption methods. Get higher nucleic acid and protein yields.

Try this simple test ...

1) Suspend your cells or tissue sample in $1 \mathrm{ml}$ of extraction solution.

2) Add an equal volume of ceramic beads (see www.biospec/beads.htm for size and type).

3) Agitate on a vortexer for 5-10 minutes (or use our MiniBeadbeater for much faster results).

Compare results with a nonagitated control.

Impressed? Visit us for more information.

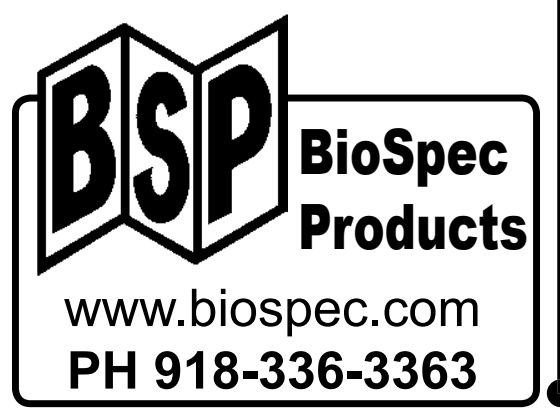

A

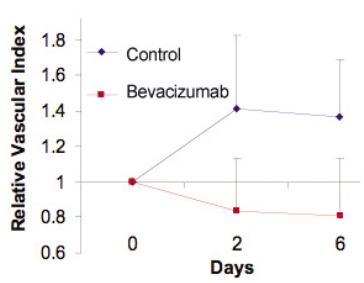

B

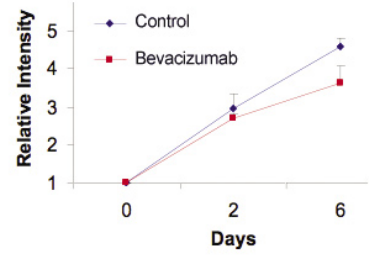

D
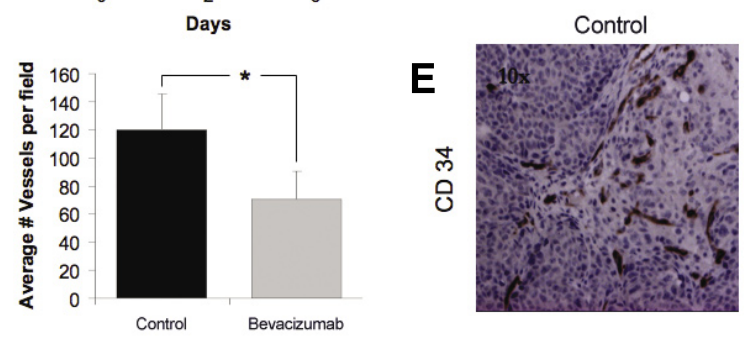

Bevacizumab Treated
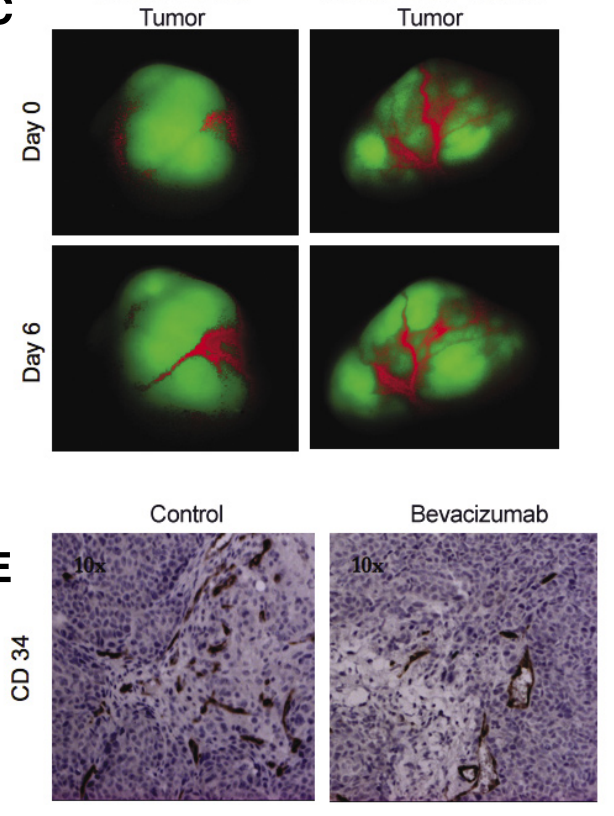

Figure 3. Therapy-induced changes in tumor vascularization can be imaged and quantitatively measured using multispectral vascular imaging. (A) In vivo vascular imaging using multispectral unmixing shows an average decrease in the relative vascular indices of tumors treated with bevacizumab (10 mg/kg, i.v., q2dx4), as compared with an average increase in the relative vascular indices of tumors treated with saline control $(n=3)$. (B) Angiosense $750 \mathrm{~nm}$ imaging of the same tumors shows an average relative increase in signal in both bevacizumab- and control-treated tumors. $(n=3)$. (C) Images of control- and bevacizumab-treated tumors before (day 0) and after (day 6) treatment. (D) Quantification of tumor vasculature using CD34 immunohistochemistry in the bevacizumab- and control-treated tumors following therapy shows a significant $(P<0.001, n=9)$ decrease in the number of blood vessels per $2.5 \times$ field of view. (E) CD34-stained images of control- and bevacizumab-treated tumors.

detected by the multispectral vascular imaging technique. Tumor vascularization was also detected via wholebody imaging in mice using the $\mathrm{CRi}$ Maestro (Figure 1D, left panel). An enlarged tumor image shows that both large and small vessels can also be detected macroscopically (Figure 1D, right panel). Unmixed spectral signatures can then be independently quantified to calculate a vascular index for each tumor (Figure 1E). In this way, tumor vascularization can be quantitatively measured and normalized for tumor size, which provides the ability to measure changes in tumor vascularization over time.

We next compared the sensitivity of multispectral unmixing to other commercially available vascular imaging probes. A mouse was given an intravenous (i.v.) injection of Angiosense $680 \mathrm{~nm}$ (Visen, Woburn, MA, USA) $(100 \mu \mathrm{L}, 13 \mu \mathrm{M}$ in PBS $)$ $1 \mathrm{~h}$ prior to anesthesia, as well as an injection of Q-tracker $800 \mathrm{~nm}$ (Invitrogen, Carlsbad, CA, USA) (100 $\mu \mathrm{L}, 0.6 \mu \mathrm{M}$ in PBS) $30 \mathrm{~min}$ prior to anesthesia. The mouse was imaged using the Nuance/AZ100 setup. First, the RFP signal was imaged using the
470/40 nm excitation and $495 \mathrm{~nm}$ longpass filters with a multispectral acquisition range of 580-800 nm (Figure 2A). Next, the Angiosense 680 $\mathrm{nm}$ probe was imaged using a 665/45 $\mathrm{nm}$ excitation and $695 \mathrm{~nm}$ longpass filters with an acquisition range of 680-950 nm (Figure 2B). Finally, the Q-tracker $800 \mathrm{~nm}$ probe was imaged using the 710/75 $\mathrm{nm}$ excitation and 750 $\mathrm{nm}$ longpass filters with an acquisition range of 680-950 nm (Figure 2C). The three images show the distinct emission spectra of the multispectral unmixing technique as well as the two vascular imaging probes. Each method detected the same tumor blood vessels, which demonstrates comparable sensitivity of the multispectral unmixing method and the two commercially available vascular imaging probes.

We then performed a proof-ofprinciple antiangiogenic therapy trial in mice with HCT116 p53-null, RFP-expressing tumors. Two weeks after tumor implantation, mice were treated with either a saline control or bevacizumab $(10 \mathrm{mg} / \mathrm{kg})$, dosed intravenously every other day for one week. Tumor vascularization was monitored in real-time with the Maestro small- 
animal imager, performing both multispectral imaging as well as imaging with the Angiosense $750 \mathrm{~nm}$ fluorescent probe. With real-time multispectral vascular imaging we detected an average relative decrease in the vascular indices of three tumors treated with bevacizumab as compared with an average relative increase in the vascular indices of three control-treated tumors (Figure 3A). With real-time imaging using Angiosense $750 \mathrm{~nm}$, we detected an average relative increase in both control- and bevacizumab-treated tumors, although the increase was slightly less in bevacizumab-treated tumors on day 6 of therapy (Figure 3B). Angiosense $750 \mathrm{~nm}$ was imaged $20 \mathrm{~min}$ after retro-orbital injection. Representative images of tumors imaged via multispectral vascular imaging before and after therapy are shown in Figure 3C. Following the bevacizumab treatment trial, the tumors were fixed in $4 \%$ paraformaldehyde, paraffin-embedded and sectioned for immunohistochemistry. Tumor sections were stained with CD34 (MEC 14.7) (Abcam, Cambridge, MA, USA) diluted 1:50 in PBT (PBS, 0.1\% BSA, 0.2\% Triton X-100). Quantification of CD34-positive blood vessels showed a significant decrease in the average number of blood vessels per $2.5 \times$ field of view in bevacizumab-treated tumors as compared with control-treated tumors $(P<$ $0.001, n=9$, Student's $t$-test) (Figure 3D). Figure 3E shows representative fields from the control-treated tumor with the greatest relative vascular index increase and the bevacizumab-treated tumor with the greatest relative vascular index decrease as measured by in vivo multispectral vascular imaging. This preclinical animal trial demonstrates that in vivo vascular imaging using multispectral unmixing is able to reliably detect subtle changes in tumor vascularization in real time, acutely following antiangiogenic therapy.

In this report we have demonstrated a novel method by which tumor vascularization can be imaged noninvasively in fluorescent tumors without the use of exogenously administered vascular imaging probes. By obviating the need for vascular probes, common problems associated with their use-including high cost, toxicity, inconsistent delivery and differential bioavailability among animals-are eliminated. Furthermore, this method completely eliminates the problem associated with blood-pooling probes of high tumor accumulation resulting from vessel leakiness. Therefore, we believe that the multispectral unmixing method described here is superior to currently used vascular imaging probes for detecting tumor vascularization as well as for monitoring dynamic changes in tumor vascularization.

\section{ACKNOWLEDGMENTS}

We thank the G.I. Morphology Core of the University of Pennsylvania, funded by the National Institutes of Health (NIH; grant no. P30 DK50306), for the use of equipment and reagents for immunohistochemistry. We also thank the Optical Imaging Core of the University of Pennsylvania School of Medicine for the use of the fluo-

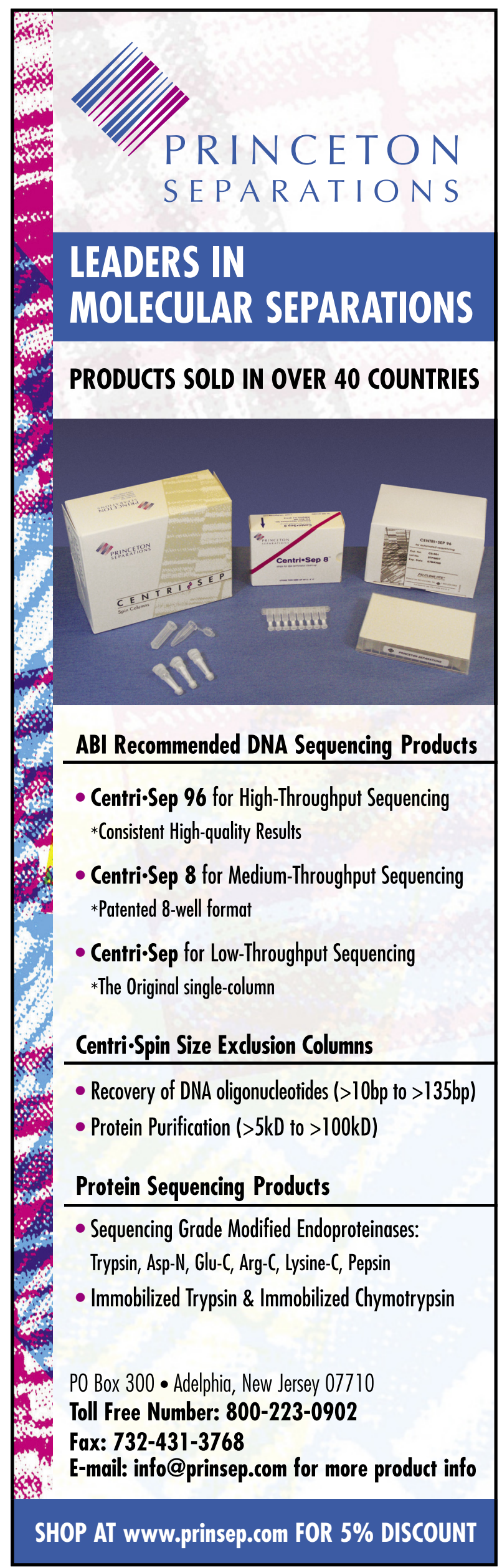




\section{Benchmarks}

rescent imagers. This work was funded by the National Institutes of Health (NIH; grant no. U54 CA105008) for The Network for Translational Research in Optical Imaging. This paper is subject to the NIH Public Access Policy.

\section{COMPETING INTERESTS STATEMENTS}

The authors declare no competing interests.

\section{REFERENCES}

1. Carmeliet, P. and R.K. Jain. 2000 Angiogenesis in cancer and other diseases. Nature 407:249-257.

2. Kerbel, R. and J. Folkman. 2002. Clinical translation of angiogenesis inhibitors. Nat. Rev. Cancer 2:727-739.

3. Lewin, M., S. Bredow, N. Sergeyev, E. Marecos, A.J. Bogdanov, and $R$. Weissleder. 1999. In vivo assessment of vascular endothelial growth factor-induced angiogenesis. Int. J. Cancer 83:798-802.
4. Montet, X., J.L. Figueiredo, H. Alencar, V. Ntziachristos, U. Mahmood, and R. Weissleder. 2007. Tomographic fluorescence imaging of tumor vascular volume in mice. Radiology 242:751-758.

5. Lee, P.J. and G.A. Peyman. 2003. Visualization of the retinal and choroidal microvasculature by fluorescent liposomes. Methods Enzymol. 373:214-233.

6. Josephson, L., M.F. Kircher, U. Mahmood, Y. Tang, and R. Weissleder. 2002. Nearinfrared fluorescent nanoparticles as combined MR/optical imaging probes. Bioconjugate Chem. 3:554-560.

7. Lewis, J.D., G. Destito, A. Zijlstra, M.J. Gonzalez, J.P. Quigley, M. Manchester, and H. Stuhlmann. 2006. Viral nanoparticles as tools for intravital vascular imaging. Nat. Med. 3:354-360.

8. Larson, D.R., W.R. Zipfel, R.M. Williams, S.W. Clark, M.P. Bruchez, F.W. Wise, and W.W. Webb. 2003. Water-soluble quantum dots for multiphoton fluorescence imaging in vivo. Science 5624:1434-1436.

9. Brown, J.M. and A.J. Giaccia. 1998. The unique physiology of solid tumors: opportunities (and problems) for cancer therapy. Cancer Res. 58:1408-1416.

10. Yuan, F., M. Leunig, S.K. Huang, D.A. Berk, D. Papahadjopoulos, and R.K. Jain. 1994. Microvascular permeability and interstitial penetration of sterically stabilized (stealth) liposomes in a human tumor xenograft. Cancer Res. 13:3352-3356.

11. Jain, R.K. 1987. Transport of molecules across tumor vasculature. Cancer Metastasis Rev. 6:559-593.

12. Bremer, C., M. Mustafa, A. Bogdanov, V. Ntziachristos, A. Petrovsky, and $R$. Weissleder. 2003. Steady-state blood volume measurements in experimental tumors with different angiogenic burdens-a study in mice. Radiology 226:214-220.

Received 24 March 2008; accepted 1 July 2008

Address correspondence to Dr. Wafik S. ElDeiry, University of Pennsylvania, School of Medicine, 437A Clinical Research Bldg., 415 Curie Blvd. Philadelphia, PA 19104. email:wafik@mail.med.upenn.edu

To purchase reprints of this article, contact: Reprints@BioTechniques.com

\section{MAESTRO IN VIVO IMAGING SYSTEMS}

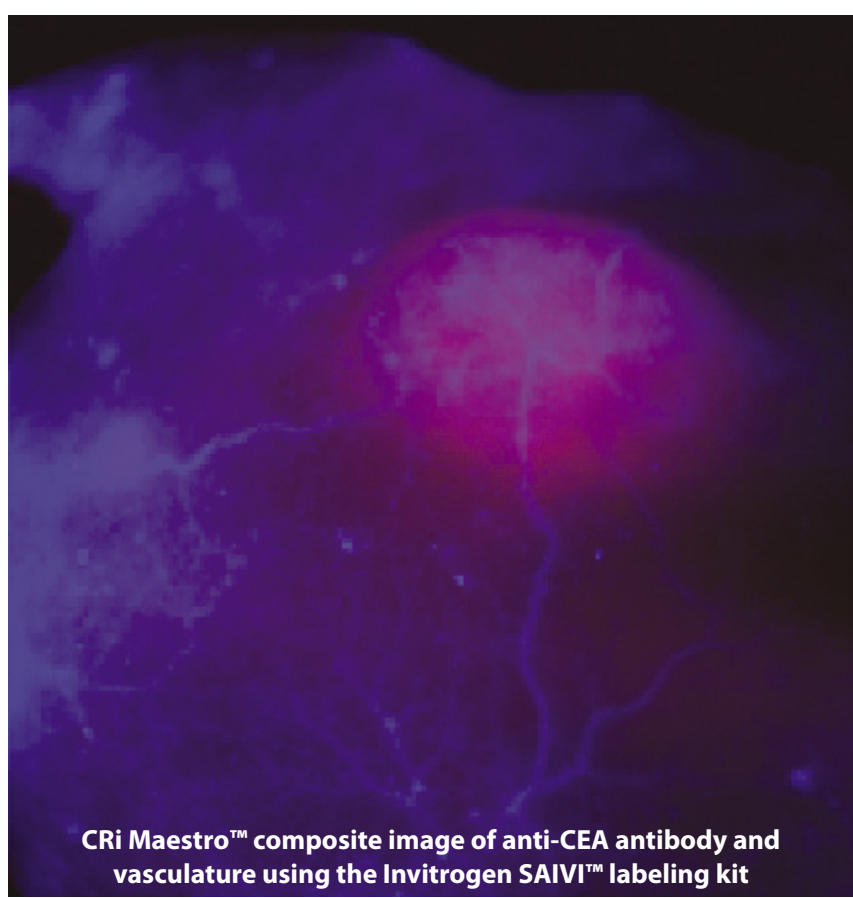

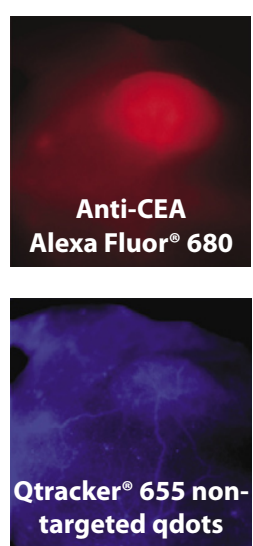

Maestro $^{\mathrm{TM}}$ composite image (left) of a fluorescent tumor (red) and vasculature (blue) in a live mouse. The individual tumor and vasculature signals can be seen above.
Call us at $1-800-383-7924$ or go to www.cri-inc.com/maestro to learn how we can enable your next breakthrough

\section{Non-Invasively Monitor Tumor Vasculature in Your Mouse Model}

\author{
CRi Maestro ${ }^{\mathrm{TM}}$ in vivo imaging systems are the most sensitive \\ fluorescence systems available: up to 300 times more sensitive \\ than monochrome
}

Quantitate more markers and resolve even closely spaced or overlapping emission signals. Conduct longitudinal studies on one animal.

Maestro systems are now available with Dynamic Contrast Enhancement $\left(D_{y} C^{\mathrm{TM}}\right)$, the powerful tool for generating alloptical anatomic maps from the same animal and dramatically reducing the time it takes to separate specific from non-specific binding

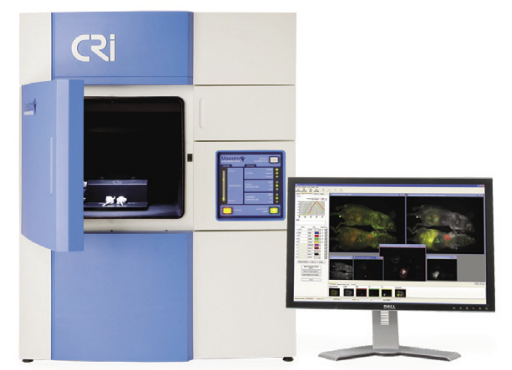

